\title{
Assessment of traditional anomalies associated with folate antagonist methotrexate in chick embryo
}

\author{
Rida Amjad ${ }^{1}$, Gulnaz Afzal ${ }^{1}$, Sajid Hameed ${ }^{2}$, Farooq Ahmad ${ }^{1 *}$, \\ Shaukat Ali ${ }^{3}$, Maimoona Nazir ${ }^{1}$, Muhammad Abdul Ghafar ${ }^{1}$, Sadaqat \\ Ali $^{1}$, Hafiz Muhammad Sajawal ${ }^{1}$ and Khola Bint Mudassar ${ }^{1}$ \\ 1. Department of Zoology, The Islamia University of Bahawalpur, Bahawalpur-Pakistan \\ 2. University College of Veterinary and Animal Sciences, The Islamia University of Bahawalpur, Bahawalpur- \\ Pakistan \\ 3. Department of Zoology, Government College University, Lahore-Pakistan \\ *Corresponding author's email: farooq.ahmad@iub.edu.pk \\ Citation \\ Rida Amjad, Gulnaz Afzal, Sajid Hameed, Farooq Ahmad, Shaukat Ali, Maimoona Nazir, Muhammad Abdul \\ Ghafar, Sadaqat Ali, Hafiz Muhammad Sajawal and Khola Bint Mudassar. Assessment of traditional anomalies \\ associated with folate antagonist methotrexate in chick embryo. Pure and Applied Biology. Vol. 9, Issue 1, \\ pp1103-1109-. http://dx.doi.org/10.19045/bspab.2020.90115

\begin{tabular}{llll}
\hline \hline Received: 19/10/2019 & Revised: 01/01/2020 & Accepted: 10/01/2020 & Online First: 25/01/2020 \\
\hline \hline
\end{tabular}

\section{Abstract}

Methotrexate (MTX) analogue of folic acid is an anti-cancerous drug that has been used from many years for treatment of important diseases like rheumatoid arthritis and ectopic pregnancy. This study was performed to evaluate the gross anomalies of chicken embryos after the exposure of MTX on different development stages. A total of 144 eggs were divided into six groups with eight eggs in each group and exposed different dose concentrations viz, control (group 1) $5 \mu \mathrm{l}$ (Group 2), $10 \mu \mathrm{l}$ (Group 3), $15 \mu \mathrm{l}$ (Group 4), $20 \mu \mathrm{l}$ (Group 5), $25 \mu \mathrm{l}$ (Group 6). Gross anomalies of chick embryo regarding embryo's body weight, body length, wings length, hind limbs length, beak length and head diameter were examined on $12^{\text {th, }} 15^{\text {th }}$ and $18^{\text {th }}$ days after incubation. The results of mean values of $12^{\text {th }}, 15^{\text {th }}$ and 18th day old chick embryos exposed to $5 \mu \mathrm{l}$ and $10 \mu \mathrm{l}$ MTX showed non-significant effect $(\mathrm{P}>0.05)$ on embryo's body weight, body length, wings length, hind limbs length, beak length and head diameter of all groups with respect to control. While MTX with concentration of $15 \mu$ l showed significant effect $(\mathrm{P}<0.05)$ on the hind limb length of chick embryo. Group 3 individuals $(15 \mu \mathrm{l})$ were found with removed hairs on the neck area, slow movement of embryo after hatching and poor feeding in chicks. Moreover, among most frequent abnormalities, hind limb lengths, body length and body weight at $15 \mu \mathrm{l}$ were found reduced in size. Group 4 and group 5 with $20 \mu \mathrm{l}$ and $25 \mu \mathrm{l}$ dose concentration respectively caused complete termination of chick embryos.

Keywords: Chick embryo; Embryonic stages; Gross abnormalities; Methotrexate

\section{Introduction}

Methotrexate (MTX), an analogue of folic acid, is an amethopterin drug having a chemotherapeutic agent in it and is being used for the treatment of rheumatoid arthritis, ectopic pregnancy, acute lymphoblastic leukaemia. This drug is also being used for the treatment of inflammatory diseases because it has the ability to affect immune signaling and on the process of DNA synthesis; both are responsible for the reduction of the inflammation process [1]. 
A serious disease, called rheumatoid arthritis, commonly found in humans is being cured by MTX. In European countries, this drug is also thought to be best treatment for another disease, called psoriasis condition. Psoriasis disease is characterized by abnormal production of skin cells due to autoimmune disorder by leaving patient's skin scaly and thick [2].

Although MTX is considered as safe medication, it induces some acute and chronic disorders. Neurotoxicity and central immune organs damages are the major side effects of MTX [3, 4]. Another abnormality caused by MTX is the renal toxicity causing vasoconstriction of arterioles, reduction in embryo mass and tarsus length $[5,6]$. Hematologic toxicity is also a problem faced after MTX administration [7] while it also affects the formation of primordial follicles [8].

Vatansever [9] described the results of MTX effect on chick neural tube in which application of this drug caused retardation in general embryonic progress and further retardation in the development of the neural system by impairing the folate metabolism at the cellular level, which ends in an open neural tube. Natekar [10] revealed in his research that MTX has the ability to induce the gross malformations in chick embryo i.e. growth retardation, beak deformity, limb deformities, short wings and sometimes immediate death.

Objective of the present study was to evaluate the gross structural anomalies regarding chick embryo length, weight, wing length, hind limb length, beak length and head diameter. Dose and time specific abnormalities after MTX exposure were investigated.

\section{Materials and methods Handling of chick eggs}

A total of 144 fertilized, fresh, White Leghorn chicken breed eggs were taken from the Govt. poultry farm Bahawalpur, Punjab, Pakistan. Eggs were distributed in the six groups viz. Group 1, Group 2, Group 3, Group 4 and Group 5 and Control group. Group names were assigned according to the dose concentration level from lower to higher viz. Group $1(5 \mu \mathrm{l})$, Group $2(10 \mu \mathrm{l})$, Group $3(15 \mu 1)$, Group 4 (20 $\mu$ l), Group 5 $(25 \mu \mathrm{l})$ and control group (non-treatment group). Eight replicates were taken in each group. Eggs were cleaned with ethanol and then kept in the incubator. Incubation of eggs started from the next day of hatching and the doses were injected to the eggs on the 5th day of incubation. For the injection purpose firstly eggs were removed from the incubator and kept in the room for two hours then again cleaned with cotton soaked with $70 \%$ ethanol. After gentle cleansing, eggs were kept in the trays which were also cleaned by $70 \%$ ethanol. For identification of all eggs, with the help of markers, eggs were marked and labeled accordingly into 6 groups. After labeling, airsacss were localized with the help of a torch and then a hole of $2 \mathrm{~mm}$ was made in the airsac of each replicate by egg driller. One $10 \mathrm{mg}$ tablet of MTX was taken and pasted into powder form properly. It was weighed again and then dissolved in the $10 \mathrm{ml}$ of distilled water. From this solution, all the injections of doses were taken directly. After injections, the eggs were sealed by paraffin wax. All eggs were placed in the room temperature for two hours and then shifted to the incubator. The first sacrifice of eggs was done on the $12^{\text {th }}$ day of incubation. On that day, all the six groups were sacrificed and measurements of all listed parameters were taken very precisely and carefully. The whole experiment method was repeated three times, only the sacrifice day was changed i.e. $12^{\text {th }}, 15^{\text {th }}$ and $18^{\text {th }}$ day of incubation respectively. From the injection day, no sacrifice was done and hatching of chicks occurred in the incubator on the $20^{\text {th }}$ day. All experimental protocols and animal handling procedures were approved by ethical review board of The Islamia University of Bahawalpur, Pakistan.

\section{Examinations of chick embryos}

Eight eggs from each group were taken out on the $12^{\text {th }}$ day, $15^{\text {th }}$ and $18^{\text {th }}$ day. After cracking the eggs, the embryo was 
separated from the egg yolk and kept in the Petri dish for proper examination. Embryo body length, body weight, head diameter, beak length, wing length and hind limbs length was measured very carefully. All measurements were taken by the standard six inches scale.

\section{Statistical analysis}

Graphpad Prism ver 5.04 (GraphPad Software, Inc.) was used for graphs and statistical analysis. All MTX-treated group's means and standard deviation values regarding all listed parameters were calculated in relation to the control group. The Bonferroni Multiple Comparative Test was used in the form of one-way ANOVA. All the dose concentrations related deformities and abnormalities were examined in relation to control and treatment groups.

\section{Results}

Effect of MTX on mortality is show in (Figure 1). Control and group 2, 3 and 4 did not any mortality with $0,5,10$ and $15 \mu \mathrm{l}$ MTX doses. However, embryos of group 5 and 6 with MTX doses of 20 and $25 \mu 1$ were terminated completely on $12^{\text {th }}$ day after incubation and hence were not used for further endpoints scored in the present study.

Effect of MTX on chick embryo (12 $2^{\text {th }}$ day) is shown in (Figure 2). The data indicated that embryo weight was reduced significantly $(\mathrm{P}<0.05)$ in all the doses as compared to control. Similarly, there was a significant difference among the three doses as well. Embryo body length was also reduced significantly $(\mathrm{P}<0.05)$ in group 2 and 3 while group was not significantly different from the control. Embryo wing length was reduced significantly $(\mathrm{P}<0.05)$ only in case of group 4, while group 2 and 3 were not significantly different as compared to control. Embryo hind limb length was reduced significantly $(\mathrm{P}<0.05)$ in group 3 and 4 as compared to control while group 1 was not significantly different from control group. There was no significant difference for Embryo beak length between control and all doses tested
( $\mathrm{P}>0.05)$. Embryo's head diameter was reduced significantly $(\mathrm{P}<0.05)$ in group 3 and 4 while group 2 was not significantly different as compared to control.

Effect of MTX on chick embryo (15 ${ }^{\text {th }}$ day) is shown in (Figure 3). It was found that embryo weight was reduced significantly in dose-dependent manner $(\mathrm{P}<0.05)$ in all doses groups as compared to control. Body length was also reduced significantly $(\mathrm{P}<0.05)$ in all the doses as compared to control. However, no significant difference was found among any treated groups. Embryo's wing length was reduced significantly $(\mathrm{P}<0.05)$ in all dose groups as compared to control $(\mathrm{P}<0.05)$. There was a no significant difference among any dose groups tested. Embryo hind limb length was reduced significantly $(\mathrm{P}<0.05)$ in group 2 and 3 while group 2 was not different as compared to control. Embryo beak length of control was not significantly different from any of the groups tested with MTX $(\mathrm{P}>0.05)$. Embryo's head diameter was reduced significantly $(\mathrm{P}<0.05)$ in group 2 and 3 but not with group 1 as compared with control. However, one way ANOVA revealed no significant difference between dose 2 and dose 3 groups but significantly reduced as compared with dose 1 group.

Effect of MTX on chick embryo ( $18^{\text {th }}$ day) is shown in (Figure 4). Our results indicated that, embryo weight was reduced significantly $(\mathrm{P}<0.05)$ in all the doses as compared to control. Among dose groups, dose 1 group was significantly different as compared to group 3 while group 2 and 3 did not differ among themselves. Embryo body length was significantly reduced in all the doses as compared to control. Embryo's wing length was also reduced significantly in all the doses as compared to control. However, the treated groups did not differ significantly among themselves. Embryo hind limb length was reduced significantly $(\mathrm{P}<0.05)$ in all the doses as compared to control. Among dose groups, group 3 was significantly different as compared to group 1 and 2 which did not differ among themselves. Embryo beak length of control 
group was not significantly different $(\mathrm{P}<0.05)$ as compared to all doses groups tested. Embryo's head diameter was also not affected $(\mathrm{P}>0.05)$ in all the doses as compared with control group.

Taken together, the results of the present study suggest that even lower doses such as $5 \mu \mathrm{l}$ (Group 2) and $10 \mu \mathrm{l}$ (group 3) caused different kind of abnormalities in chick embryo while higher dose $15 \mu 1$ (Group 4) produced major anomalies in chick embryos. Group $5(20 \mu \mathrm{l})$ and group 6 (25 $\mu \mathrm{l})$ totally terminated the developing embryo and proved as lethal doses. The eggs having an injection of this lethal dose produced a blackish, diminished and smelly developing embryo.

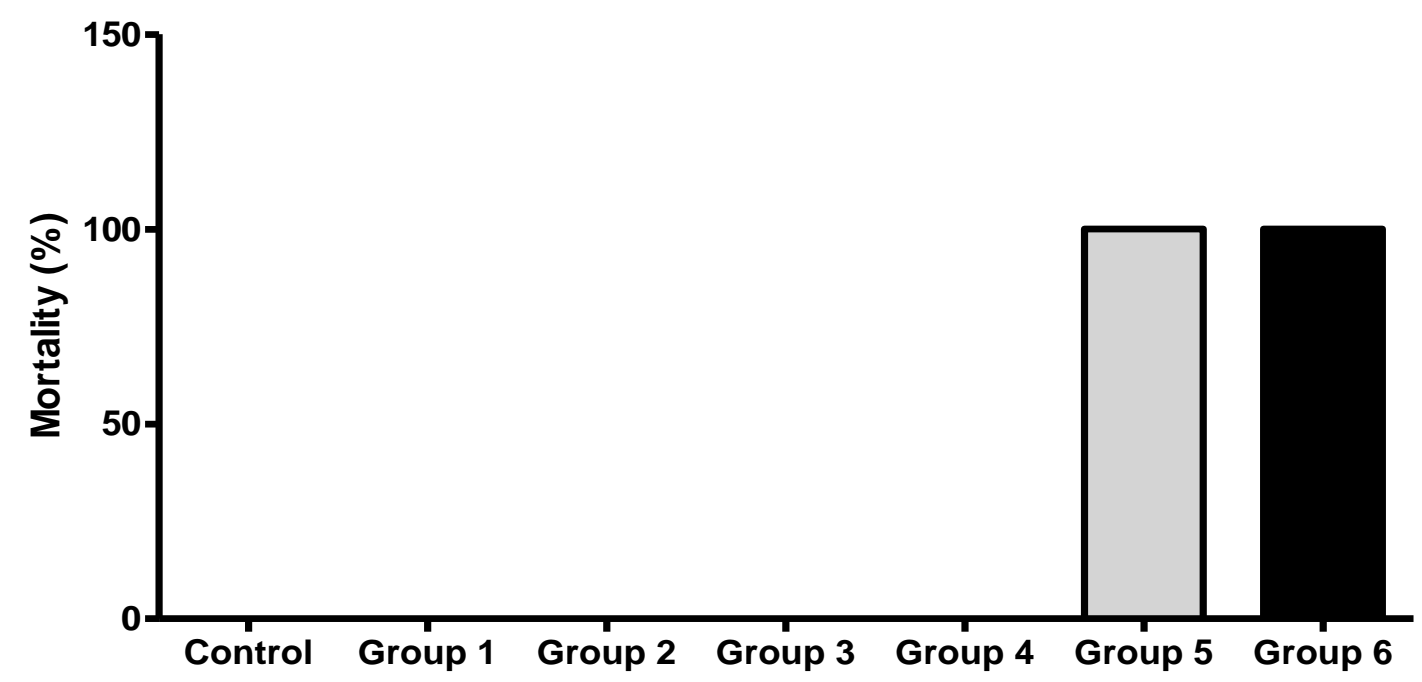

Figure 1. Effect of MTX on mortality: no mortality was observed in control group as well as Group 1, 2 and 3 having MTX dose of 5, 10 and $15 \mu$ respectively. Group 5 and 6 representing MTX doses of 20 and $25 \mu$ l were terminated completely showing $100 \%$ mortality score

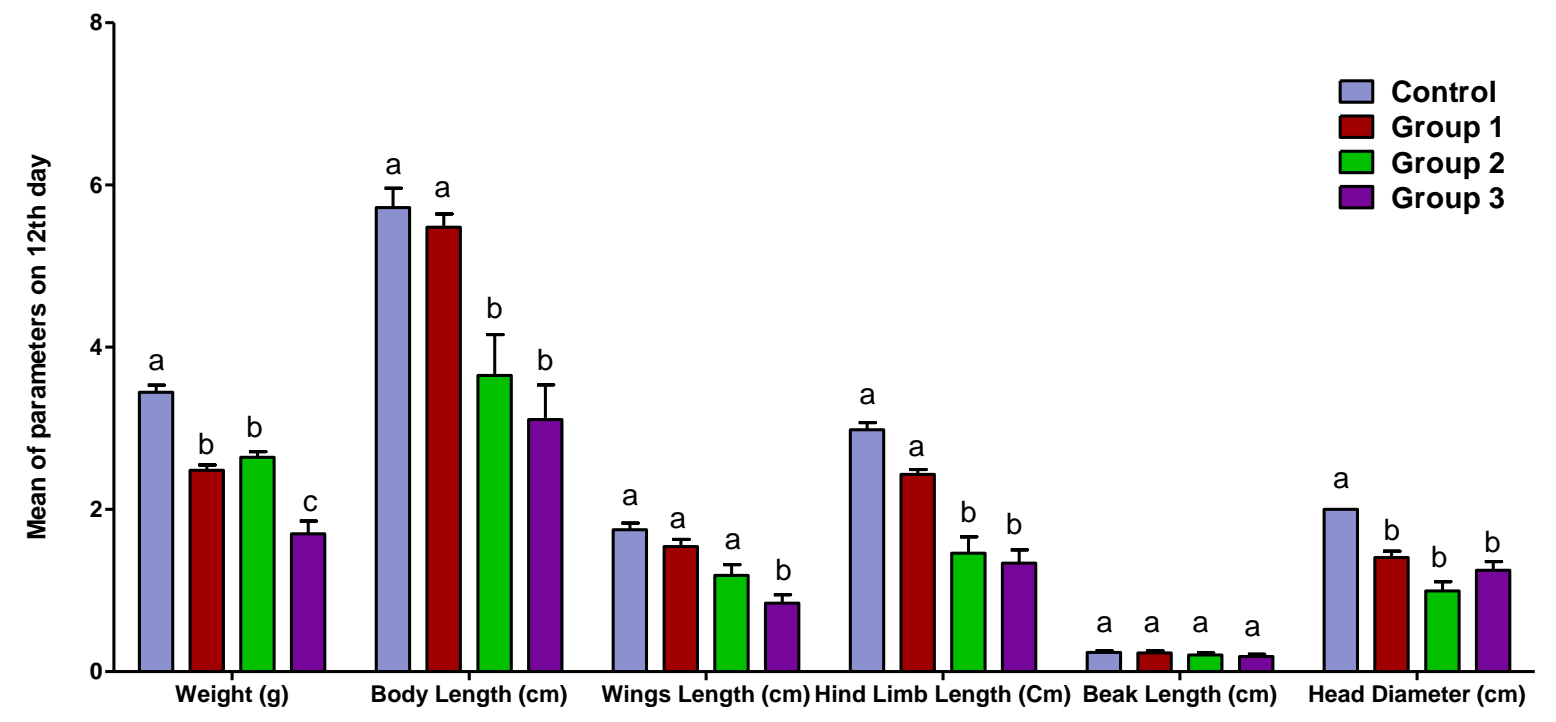

Figure 1. The Mean \pm SEM of all parameters on $12^{\text {th }}$ day embryos at different levels of MTX doses. Different superscript letters indicate significant difference $(P<0.05)$ as assessed by one-way ANOVA followed by a Bonferroni multiple comparison test 


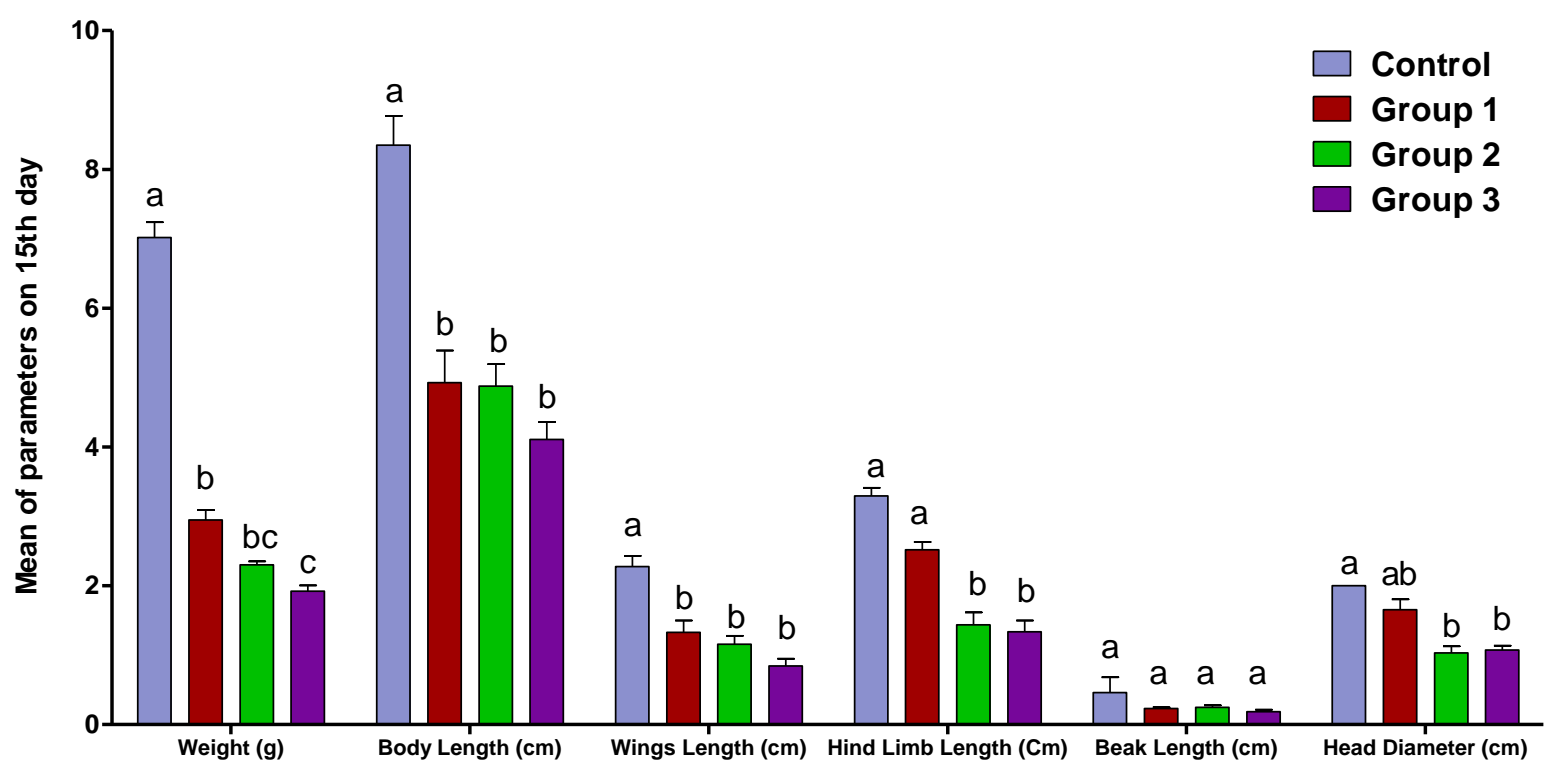

Figure 2. The Mean \pm SEM of all parameters of $15^{\text {th }}$ day embryos at different levels of MTX doses. Different superscript letters indicate significant difference $(P<0.05)$ as assessed by one-way ANOVA followed by a Bonferroni multiple comparison test

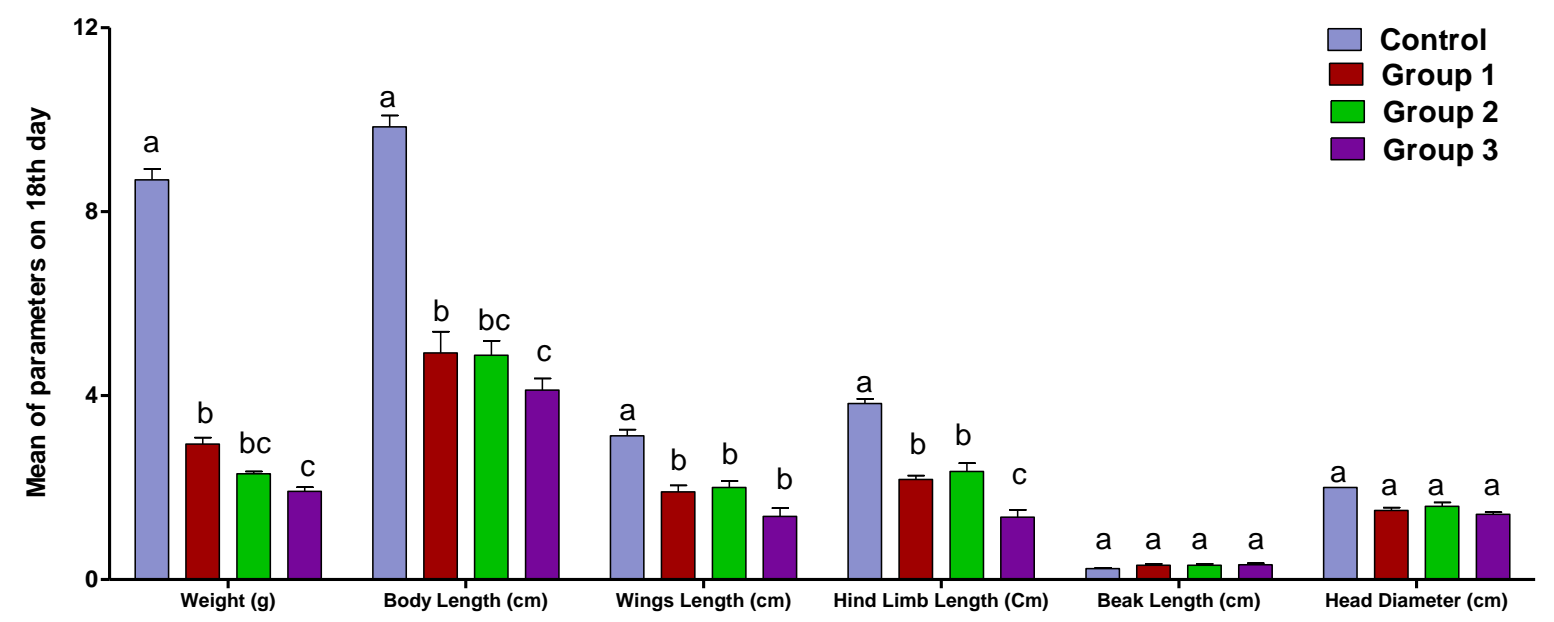

Figure 3. The Mean \pm SEM of all parameters of 18th day embryos at different levels of MTX doses. Different superscript letters indicate significant difference $(P<0.05)$ as assessed by one-way ANOVA followed by a Bonferroni multiple comparison test

\section{Discussion}

The results from the present study are supported by the findings of Natekar [10] which was conducted on chick embryo and found stunted growth, short beak, limb deformities, scanty feathers and short wings [10].
A number of studies have been conducted on the harmful effects of MTX in human morphology and anatomy. Powell and Ekert [11] conducted a study on human beings regarding the teratogenicity caused by MTX such as brain malformations i.e. Spina bifidia, anencephaly and central nervous system (CNS) anomalies. A similar 
study was also reported by conducted by Buckley, Bullaboy [12]. In yet another study by Blackburn and Alarcon [13] that MTX induced the impotence (inability to take effective action) when it was used in rheumatic arthritis.

The harmful effects of MTX have been studied in animals too. Karnofsky, Patterson [14] found that MTX delays the embryonic growth in chickens. Brewton and MacCabe [15] designed a study in chicks and resulted in limb anomalies induced by inhibition of cell division.

Species specific effects with embryo toxicity with cats and embryo toxicity and teratogenicity in rats, mice and rabbits have also been reported [16-18]. Administration of MTX in rabbits caused hydrocephalus, microphthalmia, cleft lip and palate, dysplastic vertebrae and distal limb dysplasia [18]. Studies by Wilson, Scott [19] showed the malformations were largely confined to caudal vertebrae in rats following exposure to MTX.

DeSesso and Goeringer [20] revealed that MTX injected into the rabbits caused the most common defects such as digits and limbs abnormalities. In a series of studies, malformations induced by cyclophosphamide in chick embryos were observed [21-26]. The data on the effect of MTX on other animal species is scarce, which is why results of present study become important to know the effects of this important anti cancerous drug. The major abnormalities noticed in the current study imply that teratogenic effects are species specific [27]. Further studies on the effect of MTX on chicks as well as other species might be helpful to know about efficacy and understand the mechanism of action of MTX.

\section{Conclusion}

Present study was conducted to investigate the anomalies associated with chick embryogenesis after the exposure of MTX. This drug has been familiar to human beings for its gestational teratogenic effects. Animal studies have key role in enlightening us about the mechanism involved in developmental anomalies in similar manner as in human beings. For this obvious reason, a specific maternal administration of MTX was determined during developmental stages of chick embryo and the outcome was evaluated and compared with normal embryos. Further studies are required to fully understand the mechanism behind the anomalies observed in the present study.

\section{Authors' contributions}

Conceived and designed the experiments: $\mathrm{R}$ Amjad, G Afzal, S Ali \& MA Ghafar, Performed the experiments: R Amjad, S Ali \& M Nazir, Analyzed the data: G Afzal, F Ahmad, S Ali \& R Amjad, Contributed reagents/ materials/ analysis tools: $\mathrm{R}$ Amjad, F Ahmad, HM Sajawal \& KB Mudassar, Wrote the paper: R Amjad, G Afzal, S Hameed, F Ahmad \& Shaukat Ali.

\section{References}

1. Lloyd ME, Carr M, Mcelhatton P, Hall Gm \& Hughes RA (1999). The Effects of Methotrexate on Pregnancy, Fertility and Lactation. QJM 92(10): 551-563.

2. Kozub P \& Simaljakova M (2011). Systemic Therapy of Psoriasis: Methotrexate. Bratisl Lek Listy 112(7): 390-394.

3. Cohen IJ (2017). Neurotoxicity After High-Dose Methotrexate (Mtx) Is Adequately Explained By Insufficient Folinic Acid Rescue. Cancer Chemother Pharmacol 79(6): 1057-1065.

4. Tian J, Luo D, She R, Liu T, Ding Y \& Yue Z et al. (2014). Effects of Bisphenol a on the Development of Central Immune Organs of Specific-PathogenFree Chick Embryos. Toxicol Ind Health 30(3): 199-205.

5. Hagag AA, Elgamsy MA, El-Asy Hm \& Mabrouk MM (2016). Protective Role of Silymarin on Hepatic and Renal Toxicity Induced By Mtx Based Chemotherapy In Children With Acute Lymphoblastic Leukemia. Mediterr J Hematol Infect Dis 8(1): E2016043.

6. Crump D, Chiu S \& Williams Kl (2016). Bisphenol S Alters Embryonic Viability, Development, Gallbladder Size, And Messenger Rna Expression In Chicken 
Embryos Exposed Via Egg Injection. Environ Toxicol Chem 35(6): 15411549.

7. Gaies E \& Jebabli N (2012). Methotrexate Side Effects: Review Article. J Drug Metab Toxicol 3(4).

8. Yu M, Xu Y, Li M, Li D, Lu Y \& Yu D et al. (2018). Bisphenol A Accelerates Meiotic Progression In Embryonic Chickens Via The Estrogen Receptor Beta Signaling Pathway. Gen Comp Endocrinol 259: 66-75.

9. Vatansever HS, Umur AS, Inan VS \& Selçuki M (2003). The effects of methotrexate on the development of neural tube defects in the chick embryo. Turkish J of Vet and Animal Sci 27(5): 1119-1125.

10. Natekar PE (2007). Methotrexate Induced Gross Malformations In Chick Embryos. J of Human Ecol 21(3): 223226.

11. Powell HR \& Ekert H (1971). Methotrexate-Induced Congenital Malformations. Med J Aust 2(21): 10761077.

12. Buckley Lm, Bullaboy Ca, Leichtman L \& Marquez M (1997). Multiple Congenital Anomalies Associated With Weekly Low-Dose Methotrexate Treatment Of The Mother. Arthritis Rheum 40: 971-973.

13. Blackburn Wd, JR \& Alarcon GS (1989). Impotence In Three Rheumatoid Arthritis Patients Treated With Methotrexate. Arthritis Rheum 32(10): 1341-1342.

14. Karnofsky DA, Patterson PA \& Ridgway LP (1949). Effect of Folic Acid, "4-Amino" Folic Acids and Related Substances on Growth of Chick Embryo. Proc Soc Exp Biol Med 71: 447-452.

15. Brewton RG \& Maccabe JA (1990). Studies of Methotrexate-Induced Limb Dysplasias Utilizing a 51chromium Release Assay. Teratol 41: 211-221.

16. Skalko Rg \& Gold Mp (1974). Teratogenicity of Methotrexate in Mice. Teratol 9(2): 159-163.

17. Khera Ks (1976). Evaluation of the Cat for Teratogenicity Studies. Fifteenth
Annual Meeting of the Society of Toxicology Atlanta, Georgia. Toxicol and Appl Pharmacol 149-150.

18. Jordan Rl, Wilson Jg \& Schumacher $\mathrm{Hj}$ (1977). Embryotoxicity of the Folate Antagonist Methotrexate In rats and rabbits. Teratol 15(1): 73-79.

19. Wilson Jg, Scott Wj, Ritter Ej \& Fradkin R (1979). Comparative Distribution and Embryotoxicity of Methotrexate in Pregnant Rats and Rhesus Monkeys. Teratol 19(1): 71-79.

20. Desesso Jm \& Goeringer Gc (1992). Methotrexate-Induced Developmental Toxicity In Rabbits Is Ameliorated By 1(P-Tosyl)-3,4,4-

Trimethylimidazolidine, A Functional Analog For Tetrahydrofolate-Mediated One-Carbon Transfer. Teratol 45(3): 271-283.

21. Singh S, Kar AK \& Sanyal AK (1973). Prenatal Retardation of Brain Growth Of Chick Embryos Following Cyclophosphamide Administration. Neurol India 21(3): 104-112.

22. Singh S \& Malik Mr (1976). Histological Changes in Hydrocephalic Brains of Chick Embryos Induced by Cyclophosphamide. Teratol 13(37).

23. Singh S, Sanyal Ak \& Kar AK (1974). The Effect of Cyclophosphamide on the Morphogenesis of the Cerebellum In Chick Embryos. Anat Rec 178(1): 127137.

24. Singh S, Tuli SM \& Gupta PK (1971). Skeletal Defects Induced By Cyclophosphamide (Endoxan-Asta) In Chick Embryos--Preliminary Report. Acta Orthop Scand 42(3): 217-226.

25. Singh Sag PK (1972). Lethality and Teratogenecity of Cyclophosphamide (Endoxan Asta) In Chick Embryos. Congenital Anomalies 12: 61-72.

26. Singh Sas, DN (1973). Chlorambucil Induced Malformations in Chick Embryos. $J$ of Anatomical Soc of India 22(2): 70-77.

27. Shepard TH (1979). Teratogenicity of Therapeutic Agents. Curr Probl Pediatr 10(2): 1-42. 